\title{
Combinatorialization of Sury and McLaughlin Identities and General Linear Recurrences by a Unified Approach
}

\author{
Sudip Bera \\ Department of Mathematics, Indian Institute of Science, Bangalore \\ Email: sudipbera@iisc.ac.in \\ Received:September 15, 2020, Accepted: November 16, 2020, Published: November 22, 2020 \\ The authors: Released under the CC BY-ND license (International 4.0)
}

ABSTRACT: In this article, we provide combinatorial proofs of some prior identities due to Sury and McLaughlin. We show that the solution of a general linear recurrence with constant coefficients can be interpreted as a determinant of a matrix. Moreover, we derive determinantal expressions for the Fibonacci and Lucas numbers. We prove Binet's formula for Fibonacci and Lucas numbers in a purely combinatorial way and, in the course of doing so, find a determinantal identity which we believe to be new.

Keywords: Combinatorial proof; Determinants; Digraphs; Symmetric functions; Words 2020 Mathematics Subject Classification: 05A19; 05A05; 05C30; 05C38

\section{Introduction}

This paper involves finding combinatorial proofs of some prior identities due to Sury and McLaughlin and providing a unified treatment of determinantal formulas of general linear recurrence with constant coefficients. It should be noted that combinatorial proofs are often preferred by enumerative combinatorists to more analytical ones as they frequently yield more insight into "why" a particular result is true rather than just "how" it is true $[1,5,8,12]$.

It is not always possible to find a closed form expression for an arbitrary term of a sequence defined by a recurrence relation with given initial conditions. Many well-known integer sequences, however, such as the Fibonacci and Lucas numbers and their generalizations and relatives, do admit to such a closed form. Identities concerning Fibonacci numbers and their relatives frequently can be afforded a combinatorial explanation in terms of tilings of an $n$-board [4]. Moreover, there are several known proofs of Binet's formula for the Fibonacci and Lucas numbers [2,3], some of which are combinatorial. In particular, in [3], a probabilistic proof employing random tilings of an infinite board with squares and dominoes is given for Binet's formula and its generalization to arbitrary initial conditions. See also the related paper [10] where infinite tilings are used to explain some reciprocal Fibonacci number relations. Here, we demonstrate that the solution of a general linear recurrence with constant coefficients can be expressed as a determinant in a purely combinatorial way and particularizing our results yields the Binet formulas for the Fibonacci and Lucas numbers. Our combinatorial approach also implies some identities due to Sury and McLaughlin.

Let us briefly summarize the content of this paper. In Section 2, we give a bijective proof of an identity regarding elementary and homogeneous symmetric polynomials and, as a corollary, one gets bijective proofs of some identities of Sury and McLaughlin. In Section 3, we prove that the solution of a general linear recurrence with constant coefficients can be interpreted as a determinant of some matrix. We prove Binet's formula for Fibonacci numbers in a combinatorial way. In Section 4, we derive a new combinatorial identity and, as a corollary, we get Binet's formula for the Lucas numbers. 


\section{Combinatorial Explanation of Sury and McLaughlin Identities}

In this section, we give combinatorial proofs of some identities due to Sury and McLaughlin. Before starting, let us review some necessary background. For further details, we refer the reader to [6,9]. Let $A=\left(a_{i j}\right)$ denote an $n \times n$ matrix. We associate with $A$ a weighted digraph $D(A)$ whose vertex set is $[n]=\{1,2, \ldots, n\}$ such that, for each ordered pair $(i, j)$, there is an edge directed from $i$ to $j$ with weight $a_{i j}$. A linear subdigraph (LSD) $L$ of $D(A)$ is a spanning collection of pairwise vertex-disjoint cycles. A loop around a single vertex is also considered to be a cycle. The weight of a linear subdigraph $L$, written as $w(L)$, is the product of the weights of all its cycles. The weight of a cycle is the product of the weights of all its edges. The length of a cycle is the number of edges present in that cycle. The number of cycles contained in $L$ is denoted by $c(L)$. Now the cycledecomposition of permutations yields the following description of $\operatorname{det}(A)$, namely, $\operatorname{det}(A)=\sum_{L}(-1)^{n-c(L)} w(L)$, where the summation runs over all linear subdigraphs $L$ of $D(A)$.

A partition $\lambda$ of a positive integer $m$ is a weakly decreasing finite sequence $\left(\lambda_{1}, \ldots, \lambda_{r}\right)$ of non-negative integers such that $\sum_{i=1}^{r} \lambda_{i}=m$. For $n$ variables $x_{1}, \ldots, x_{n}$, the elementary symmetric polynomial $e_{k}\left(x_{1}, \ldots, x_{n}\right)$ (in short, $e_{k}$ ) of degree $k \geq 0$ is defined as

$$
e_{k}\left(x_{1}, \ldots, x_{n}\right):=\sum_{1 \leq j_{1}<j_{2}<\cdots<j_{k} \leq n} x_{j_{1}} x_{j_{2}} \cdots x_{j_{k}} \text {, with } e_{0}=1 .
$$

The $n$-variable complete homogeneous symmetric polynomial $h_{k}\left(x_{1}, \ldots, x_{n}\right)$ (in short, $\left.h_{k}\right)$ of degree $k \geq 0$ is the sum of all monomials of total degree $k$. Formally,

$$
h_{k}\left(x_{1}, \ldots, x_{n}\right):=\sum_{1 \leq j_{1} \leq j_{2} \leq \cdots \leq j_{k} \leq n} x_{j_{1}} x_{j_{2}} \cdots x_{j_{k}} \text {, with } h_{0}=1 .
$$

Let $\lambda=\left(\lambda_{1}, \ldots, \lambda_{\ell}\right)$ be a partition and $\delta=(\ell-1, \ell-2, \ldots, 1,0)$, where $\lambda_{1} \geq \cdots \geq \lambda_{\ell}$ and each $\lambda_{j}$ is a non-negative integer. Then the function

$$
a_{\lambda+\delta}\left(x_{1}, \ldots, x_{\ell}\right)=\operatorname{det}\left(\begin{array}{cccc}
x_{1}^{\lambda_{1}+\ell-1} & x_{2}^{\lambda_{1}+\ell-1} & \cdots & x_{\ell}^{\lambda_{1}+\ell-1} \\
x_{1}^{\lambda_{2}+\ell-2} & x_{2}^{\lambda_{2}+\ell-2} & \cdots & x_{\ell}^{\lambda_{2}+\ell-2} \\
\vdots & \vdots & \ddots & \vdots \\
x_{1}^{\lambda_{\ell}} & x_{2}^{\lambda_{\ell}} & \cdots & x_{\ell}^{\lambda_{\ell}}
\end{array}\right)
$$

is an alternating polynomial. The Schur polynomial, denoted by $s_{\lambda}\left(x_{1}, \ldots, x_{\ell}\right)$, is defined as the ratio

$$
\frac{a_{\lambda+\delta}\left(x_{1}, \ldots, x_{\ell}\right)}{a_{\delta}\left(x_{1}, \ldots, x_{\ell}\right)}
$$

Let $e_{1}, \ldots, e_{m}$ be the elementary symmetric polynomials in the variables $x_{1}, \ldots, x_{n}$. We consider the following $m \times m$ matrix

$$
E\left(e_{1}, \ldots, e_{m}\right)=\left(\begin{array}{cccccc}
e_{1} & e_{2} & e_{3} & \cdots & e_{m-1} & e_{m} \\
1 & e_{1} & e_{2} & \cdots & e_{m-2} & e_{m-1} \\
0 & 1 & e_{1} & \cdots & e_{m-3} & e_{m-2} \\
\vdots & \vdots & \vdots & \ddots & \vdots & \vdots \\
0 & 0 & 0 & \cdots & e_{1} & e_{2} \\
0 & 0 & 0 & \cdots & 1 & e_{1}
\end{array}\right) \text {. }
$$

Theorem 2.1 (Theorem 4.5.1[9]). Let $e_{1}, \ldots, e_{m}$ be the elementary symmetric polynomials in the variables $x_{1}, \ldots, x_{n}$. Let $E\left(e_{1}, \ldots, e_{m}\right)$ be the $m \times m$ matrix defined as $(1)$. Then $\operatorname{det}\left(E\left(e_{1}, \ldots, e_{m}\right)\right)=h_{m}\left(x_{1}, \ldots, x_{n}\right)$.

Proof. We prove the theorem in the case $m=5, n=3$ (similarly, one can show the general case). Regard $W\left(x_{1}, x_{2}, x_{3}\right)=\left\{x_{1}, x_{2}, x_{3}\right\}$ as the set of letters. The free monoid $W\left(x_{1}, x_{2}, x_{3}\right)^{*}$ is the set of all finite sequences (including the empty sequence, denoted by 1 ) of elements of $W\left(x_{1}, x_{2}, x_{3}\right)$, usually called linear words, with the operation of concatenation. Construct an algebra from $W\left(x_{1}, x_{2}, x_{3}\right)^{*}$ by taking formal sums of elements of 


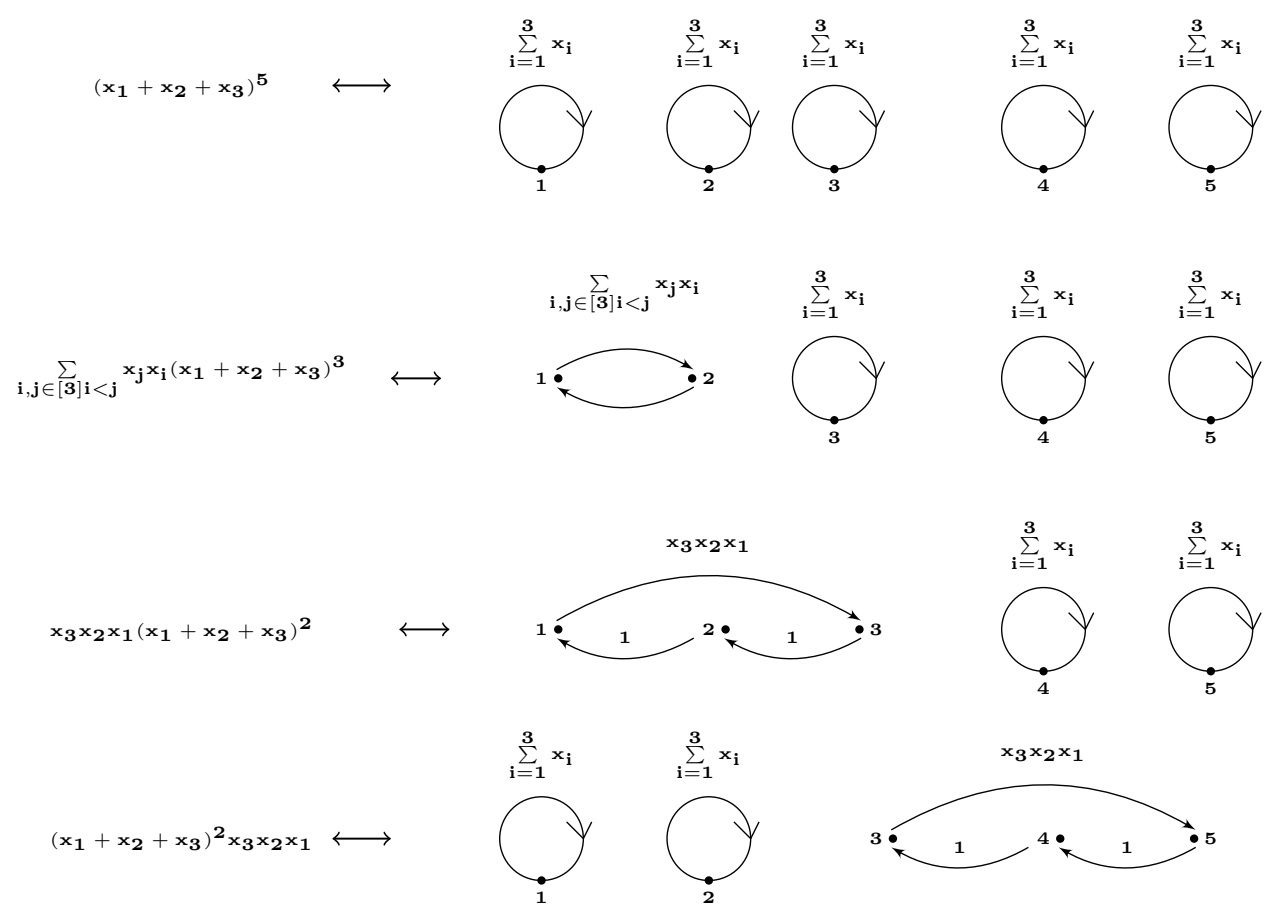

Figure 1: The numbers appearing along the edges in the above diagram are the weights of the corresponding edges. The left-hand side of the figure contains some terms of the PIE expression.

$W\left(x_{1}, x_{2}, x_{3}\right)$ with coefficient in $\mathbb{Z}$ and extending the multiplication by the usual distributive law. For example, in this algebra, $\left(x_{1}+x_{2}+x_{3}\right)\left(x_{1}+x_{2}+x_{3}\right)=x_{1} x_{1}+x_{1} x_{2}+x_{1} x_{3}+x_{2} x_{1}+x_{2} x_{2}+x_{2} x_{3}+x_{3} x_{1}+x_{3} x_{2}+x_{3} x_{3}$ and $\left(1+x_{3}\right) x_{2}=x_{2}+x_{3} x_{2}$. Note that $\left(x_{1}+x_{2}+x_{3}\right)\left(x_{1}+x_{2}+x_{3}\right)$ is usually written as $\left(x_{1}+x_{2}+x_{3}\right)^{2}$, etc.

Let $W_{L}$ be the sum of all linear words in $W\left(x_{1}, x_{2}, x_{3}\right)^{*}$ of length 5 , where the letter $x_{2}$ does not occur just before the letter $x_{1}$ and the letter $x_{3}$ does not occur just before the letters $x_{1}$ and $x_{2}$, i.e., $x_{2} x_{1}, x_{3} x_{1}$ and $x_{3} x_{2}$ do not occur in the words as a consecutive pair. So,

$$
W_{L}=x_{1} x_{1} x_{1} x_{1} x_{1}+x_{1} x_{1} x_{1} x_{1} x_{2}+\cdots+x_{2} x_{2} x_{2} x_{2} x_{2}+x_{1} x_{1} x_{1} x_{1} x_{3}+\cdots+x_{3} x_{3} x_{3} x_{3} x_{3} .
$$

Now we compute $W_{L}$ by using the Principle of Inclusion and Exclusion (PIE) rule.

The sum of all possible words of length 5 is $\left(x_{1}+x_{2}+x_{3}\right)^{5}$. This can be written as

$$
\left(x_{1}+x_{2}+x_{3}\right)\left(x_{1}+x_{2}+x_{3}\right)\left(x_{1}+x_{2}+x_{3}\right)\left(x_{1}+x_{2}+x_{3}\right)\left(x_{1}+x_{2}+x_{3}\right) .
$$

The sum of all possible words of length 5 , where there is an occurrence of $x_{2} x_{1}$ at least as the first two letters, is $x_{2} x_{1}\left(x_{1}+x_{2}+x_{3}\right)^{3}$. Similarly, the sum of all possible words of length 5 , where there is an occurrence of $x_{3} x_{2}$ and $x_{3} x_{1}$ at least as the first two letters, is given by $x_{3} x_{2}\left(x_{1}+x_{2}+x_{3}\right)^{3}$ and $x_{3} x_{1}\left(x_{1}+x_{2}+x_{3}\right)^{3}$, respectively. The sum of all possible words of length 5, where there is an occurrence of $x_{3} x_{2} x_{1}$ at least as the first three letters, is $x_{3} x_{2} x_{1}\left(x_{1}+x_{2}+x_{3}\right)^{2}$. Proceeding this way, and using the PIE rule, we get

$$
\begin{aligned}
W_{L}= & \left(x_{1}+x_{2}+x_{3}\right)^{5}-x_{2} x_{1}\left(x_{1}+x_{2}+x_{3}\right)^{3}-x_{3} x_{1}\left(x_{1}+x_{2}+x_{3}\right)^{3}-x_{3} x_{2}\left(x_{1}+x_{2}+x_{3}\right)^{3}- \\
& \left(x_{1}+x_{2}+x_{3}\right) x_{2} x_{1}\left(x_{1}+x_{2}+x_{3}\right)^{2}-\left(x_{1}+x_{2}+x_{3}\right) x_{3} x_{1}\left(x_{1}+x_{2}+x_{3}\right)^{2}- \\
& \left(x_{1}+x_{2}+x_{3}\right) x_{3} x_{2}\left(x_{1}+x_{2}+x_{3}\right)^{2}+\cdots+x_{3} x_{2} x_{1}\left(x_{1}+x_{2}+x_{3}\right)^{2}+ \\
& \left(x_{1}+x_{2}+x_{3}\right) x_{3} x_{2} x_{1}\left(x_{1}+x_{2}+x_{3}\right)+\left(x_{1}+x_{2}+x_{3}\right)^{2} x_{3} x_{2} x_{1} .
\end{aligned}
$$

Now the terms appearing in the above PIE expression of $W_{L}$ are $\left(x_{1}+x_{2}+x_{3}\right)^{5}, x_{2} x_{1}\left(x_{1}+x_{2}+x_{3}\right)^{3}, x_{3} x_{1}\left(x_{1}+\right.$ $\left.x_{2}+x_{3}\right)^{3}, x_{3} x_{2}\left(x_{1}+x_{2}+x_{3}\right)^{3}, \ldots, x_{3} x_{2} x_{1}\left(x_{1}+x_{2}+x_{3}\right)^{2},\left(x_{1}+x_{2}+x_{3}\right) x_{3} x_{2} x_{1}\left(x_{1}+x_{2}+x_{3}\right),\left(x_{1}+x_{2}+x_{3}\right)^{2} x_{3} x_{2} x_{1}$, and the signs of the corresponding terms are $+,-,-,-, \ldots,+,+,+$, respectively. We define the weight of a word $w$ in $W\left(x_{1}, x_{2}, x_{3}\right)^{*}$ as the homomorphic image $f(w)$, where $f: W\left(x_{1}, x_{2}, x_{3}\right)^{*} \rightarrow \mathbb{Z}\left[x_{1}, x_{2}, x_{3}\right]$ is a ring homomorphism, with $f\left(x_{1}\right)=x_{1}, f\left(x_{2}\right)=x_{2}$ and $f\left(x_{3}\right)=x_{3}$. Figure 1 illustrates the sign- and 
weight-preserving bijection between the terms appearing in the PIE expression and the linear subdigraphs of $D\left(E\left(e_{1}, \ldots, e_{m}\right)\right)$. What is really meant by this is that the weight of the linear subdigraph on the right side of Figure 1 is the image of the corresponding word on the left, under the ring homomorphism $f: W\left(x_{1}, x_{2}, x_{3}\right)^{*} \rightarrow$ $\mathbb{Z}\left[x_{1}, x_{2}, x_{3}\right]$.

Using the same combinatorial model as in the proof above, one can rederive some interesting identities of Sury and McLaughlin [7,11]. Note that the original proofs of those identities involve, to some extent, cumbersome algebraic manipulations, whereas our approach is purely combinatorial.

Corollary 2.1 (Theorem $1[7]$ ). Let $x_{1}, \ldots, x_{k}$ be independent variables. Let $e_{1}, \ldots, e_{k}$ denote the elementary symmetric polynomials in the $x_{i}$ of degrees $1, \ldots, k$ respectively. Then in the polynomial ring $K\left[x_{1}, \ldots, x_{k}\right]$, where $K$ is a field of characteristic 0 , one has the following identity for each $n \geq 1$ :

$$
\begin{aligned}
& \sum_{r_{1}+r_{2}+\cdots+r_{k}=n, r_{i} \geq 0} x_{1}^{r_{1}} x_{2}^{r_{2}} \cdots x_{k}^{r_{k}} \\
= & \sum_{2 i_{2}+3 i_{3}+\cdots+k i_{k} \leq n} c\left(i_{2}, \ldots, i_{k}, n\right) e_{1}^{n-2 i_{2}-3 i_{3}-\cdots-k i_{k}} \times\left(-e_{2}\right)^{i_{2}} \times e_{3}^{i_{3}} \times \cdots \times\left((-1)^{k-1} e_{k}\right)^{i_{k}},
\end{aligned}
$$

where

$$
c\left(i_{2}, \ldots, i_{k}, n\right)=\frac{\left(n-i_{2}-2 i_{3}-\cdots-(k-1) i_{k}\right) !}{\left.i_{2} ! \cdots i_{k} !\left(n-2 i_{2}-3 i_{3}-\cdots-k i_{k}\right)\right) !} .
$$

Proof. We consider the $n \times n$ matrix

$$
E\left(e_{1}, \ldots, e_{k}, 0, \ldots, 0\right)=\left(\begin{array}{ccccccccc}
e_{1} & e_{2} & e_{3} & \cdots & e_{k} & 0 & \cdots & 0 & 0 \\
1 & e_{1} & e_{2} & \cdots & e_{k-1} & e_{k} & \cdots & 0 & 0 \\
0 & 1 & e_{1} & \cdots & e_{k-2} & e_{k-1} & \cdots & 0 & 0 \\
\vdots & \vdots & \vdots & \ddots & \vdots & \vdots & \ddots & \vdots & \vdots \\
0 & 0 & 0 & \cdots & e_{1} & e_{2} & \cdots & e_{n-k} & e_{n-k+1} \\
0 & 0 & 0 & \cdots & 1 & e_{1} & \cdots & e_{n-k-1} & e_{n-k} \\
\vdots & \vdots & \vdots & \ddots & \vdots & \vdots & \ddots & \vdots & \vdots \\
0 & 0 & 0 & \cdots & 0 & 0 & \cdots & e_{1} & e_{2} \\
0 & 0 & 0 & \cdots & 0 & 0 & \cdots & 1 & e_{1}
\end{array}\right) .
$$

By Theorem 2.1, we get

$$
\operatorname{det}\left(E\left(e_{1}, \ldots, e_{k}, 0, \ldots, 0\right)\right)=\sum_{r_{1}+r_{2}+\cdots+r_{k}=n, r_{i} \geq 0} x_{1}^{r_{1}} x_{2}^{r_{2}} \cdots x_{k}^{r_{k}} .
$$

We know that

$$
\operatorname{det}\left(E\left(e_{1}, \ldots, e_{k}, 0, \ldots, 0\right)\right)=\sum_{L}(-1)^{n-c(L)} w(L),
$$

where the summation runs over all LSD $L$ of the digraph $D\left(E\left(e_{1}, \ldots, e_{k}, 0, \ldots, 0\right)\right)$. Clearly, each LSD in the digraph $D\left(E\left(e_{1}, \ldots, e_{k}, 0, \ldots, 0\right)\right)$ contains cycles of length at most $k$. Suppose $L$ is an arbitrary LSD and $L$ contains $i_{t}$ cycles of length $t(t=2,3, \ldots, k)$. Then $L$ contains $\left(n-2 i_{2}-3 i_{3}-\cdots-k i_{k}\right)$ loops. Note $(-1)^{n-c(L)}=(-1)^{i_{2}+2 i_{3}+3 i_{4}+\cdots+(k-1) i_{k}}$. The weight of the LSD $L$ is

$$
e_{1}^{n-2 i_{2}-3 i_{3}-\cdots-k i_{k}} \times\left(e_{2}\right)^{i_{2}} \times e_{3}^{i_{3}} \times \cdots \times\left(e_{k}\right)^{i_{k}},
$$

and

$$
c\left(i_{2}, \ldots, i_{k}, n\right)=\frac{\left(n-i_{2}-2 i_{3}-\cdots-(k-1) i_{k}\right) !}{\left.i_{2} ! \cdots i_{k} !\left(n-2 i_{2}-3 i_{3}-\cdots-k i_{k}\right)\right) !}
$$

is the number of $L$ containing $n-2 i_{2}-3 i_{3}-\cdots-k i_{k}$ loops and $i_{t}(t=2,3, \ldots, k)$ cycles of length $t$. Upon considering the weight and sign of each $L$ in Equation (3), we get

$$
\begin{aligned}
& \operatorname{det}\left(E\left(e_{1}, \ldots, e_{k}, 0, \ldots, 0\right)\right) \\
& \quad=\sum_{2 i_{2}+3 i_{3}+\cdots+k i_{k} \leq n} c\left(i_{2}, \ldots, i_{k}, n\right) e_{1}^{n-2 i_{2}-3 i_{3}-\cdots-k i_{k}} \times\left(-e_{2}\right)^{i_{2}} \times e_{3}^{i_{3}} \times \cdots \times\left((-1)^{k-1} e_{k}\right)^{i_{k}},
\end{aligned}
$$

and hence the identity. 
Corollary 2.2 (Theorem $3[7]$ ). Let $n$ be a positive integer and $x, y, z$ be indeterminates. Then

$$
\begin{aligned}
& \sum_{2 i+3 j \leq n}(-1)^{i}\left(\begin{array}{c}
i+j \\
j
\end{array}\right)\left(\begin{array}{c}
n-i-2 j \\
i+j
\end{array}\right)(x+y+z)^{n-2 i-3 j}(x y+y z+z x)^{i}(x y z)^{j} \\
= & \frac{x y\left(x^{n+1}-y^{n+1}\right)-x z\left(x^{n+1}-z^{n+1}\right)+y z\left(y^{n+1}-z^{n+1}\right)}{(x-y)(x-z)(y-z)} .
\end{aligned}
$$

Proof. Let $\lambda=(n)$ denote the partition of $n$ consisting of a single part. Then the right-hand side of this identity is the Schur polynomial

$$
\begin{gathered}
s_{\lambda}(x, y, z)=\frac{a_{\lambda+\delta}(x, y, z)}{a_{\delta}(x, y, z)} \text {, where } \\
a_{\lambda+\delta}(x, y, z)=\operatorname{det}\left(\begin{array}{ccc}
x^{n+2} & y^{n+2} & z^{n+2} \\
x & y & z \\
1 & 1 & 1
\end{array}\right) \text { and } a_{\delta}(x, y, z)=\operatorname{det}\left(\begin{array}{ccc}
x^{2} & y^{2} & z^{2} \\
x & y & z \\
1 & 1 & 1
\end{array}\right) .
\end{gathered}
$$

So for the partition $\lambda$, we have that $s_{\lambda}(x, y, z)$ is the complete homogeneous polynomial of degree $n$ in the variables $x, y, z$. Thus, by Theorem 2.1,

$$
h_{n}(x, y, z)=\operatorname{det}\left(\begin{array}{cccccc}
e_{1} & e_{2} & e_{3} & \cdots & 0 & 0 \\
1 & e_{1} & e_{2} & \cdots & 0 & 0 \\
0 & 1 & e_{1} & \cdots & 0 & 0 \\
\vdots & \vdots & \vdots & \ddots & \vdots & \vdots \\
0 & 0 & 0 & \cdots & e_{1} & e_{2} \\
0 & 0 & 0 & \cdots & 1 & e_{1}
\end{array}\right)=\sum_{L}(-1)^{n-c(L)} w(L)
$$

where $e_{1}, e_{2}, e_{3}$ are the elementary symmetric polynomials in the variables $x, y, z$ and the order of the matrix is $n$. Now applying the same argument used in the proof of Corollary 2.1, we get the desired identity.

Corollary 2.3. Let $x, y$ be indeterminates. Then the following polynomial identity holds:

$$
\sum_{0 \leq i \leq n / 2}(-1)^{i}\left(\begin{array}{c}
n-i \\
i
\end{array}\right)(x+y)^{n-2 i}(x y)^{i}=x^{n}+x^{n-1} y+\cdots+x y^{n-1}+y^{n} .
$$

Proof. Clearly, the right-hand side of the above identity is the complete homogeneous polynomial $h_{n}(x, y)$ of degree $n$. By Theorem 2.1,

$$
\operatorname{det}\left(\begin{array}{cccccc}
x+y & x y & 0 & \cdots & 0 & 0 \\
1 & x+y & x y & \cdots & 0 & 0 \\
0 & 1 & x+y & \cdots & 0 & 0 \\
\vdots & \vdots & \vdots & \ddots & \vdots & \vdots \\
0 & 0 & 0 & \cdots & x+y & x y \\
0 & 0 & 0 & \cdots & 1 & x+y
\end{array}\right)=h_{n}(x, y)
$$

where the matrix is of order $n$. Also, we have

$$
\operatorname{det}\left(\begin{array}{cccccc}
x+y & x y & 0 & \cdots & 0 & 0 \\
1 & x+y & x y & \cdots & 0 & 0 \\
0 & 1 & x+y & \cdots & 0 & 0 \\
\vdots & \vdots & \vdots & \ddots & \vdots & \vdots \\
0 & 0 & 0 & \cdots & x+y & x y \\
0 & 0 & 0 & \cdots & 1 & x+y
\end{array}\right)=\sum_{L}(-1)^{n-c(L)} w(L)
$$

where the summation runs over all linear subdigraphs $L$. Proceeding now as in the proof of Corollary 2.1 above, one obtains the identity. 


\section{Determinantal Interpretation of General Linear Re- currences}

In this section, we prove that the solution of a general linear recurrence with constant coefficients can be expressed as the determinant of a suitable matrix.

Definition 3.1. Let $c_{1}, c_{2}, \ldots, c_{r}$ be complex numbers. Let $u_{n}$ be the sequence defined by the $r$-th order linear recurrence

$$
u_{n}=c_{1} u_{n-1}+c_{2} u_{n-2}+\cdots+c_{r} u_{n-r}, \quad n \geq 1,
$$

with initial conditions $u_{0}=1$ and $u_{j}=0$ for $j<0$.

There is a more or less a well-known combinatorial interpretation of general linear recurrences (see [4]). In fact, $u_{n}$ is the sum of weights of all tilings of a board of length $n$ with tiles of length at most $r$, where for each $i(1 \leq i \leq r)$, the weight of each tile of length $i$ is $c_{i}$ and the weight of a tiling is the product of the weights of all its tiles. Now we consider the following $n \times n$ matrix

$$
C=\left(\begin{array}{ccccccccc}
c_{1} & -c_{2} & c_{3} & \cdots & (-1)^{r+1} c_{r} & 0 & \cdots & 0 & 0 \\
1 & c_{1} & -c_{2} & \cdots & (-1)^{r} c_{r-1} & (-1)^{r+1} c_{r} & \cdots & 0 & 0 \\
0 & 1 & c_{1} & \cdots & (-1)^{r-1} c_{r-2} & (-1)^{r} c_{r-1} & \cdots & 0 & 0 \\
\vdots & \vdots & \vdots & \ddots & \vdots & \vdots & \ddots & \vdots & \vdots \\
0 & 0 & 0 & \cdots & c_{1} & -c_{2} & \cdots & (-1)^{n-r+1} c_{n-r} & (-1)^{n-r+2} c_{n-r+1} \\
0 & 0 & 0 & \cdots & 1 & c_{1} & \cdots & (-1)^{n-r} c_{n-r-1} & (-1)^{n-r+1} c_{n-r} \\
\vdots & \vdots & \vdots & \ddots & \vdots & \vdots & \ddots & \vdots & \vdots \\
0 & 0 & 0 & \cdots & 0 & 0 & \cdots & c_{1} & -c_{2} \\
0 & 0 & 0 & \cdots & 0 & 0 & \cdots & 1 & c_{1}
\end{array}\right) .
$$

Theorem 3.1. Let $u_{n}(n \geq 1)$ be a general linear recurrence defined as (3.1). Then $u_{n}=\operatorname{det}(C)$.

Proof. To prove this result, we use the combinatorial interpretation of $u_{n}$ (stated above) and the fact $\operatorname{det}(C)=$ $\sum_{L}(-1)^{n-c(L)} w(L)$, where the summation runs over all LSD $L$ in $D(C)$ (as in Section 2). In fact, we demonstrate a sign- and weight-preserving bijection between tilings of the $n$-board and the linear subdigraphs of $D(C)$. Let $\tau$ be a tiling of the $n$-board and suppose $\tau$ contains tiles of length at most $r$. Assume for each fixed $i \in[r]$ that $\tau$ contains $t_{i}$ tiles $\tau_{i 1}, \ldots, \tau_{i t_{i}}$ of length $i$. For fixed $j \in\left[t_{i}\right]$, suppose the tile $\tau_{i j}$ occupies the positions $k_{i j},\left(k_{i j}+1\right), \ldots,\left(k_{i j}+i-1\right)$, where $k_{i j} \in[n-i+1]$. (For example, the left side of Figure 2 contains two tiles of lengths 2 and 3 , which occupy the positions 1,2 , and 3,4,5, respectively.) For this tiling, we choose the LSD $L_{\tau}$ containing $t_{i}$ cycles $C_{i 1}, \ldots, C_{i t_{i}}$ each of length $i$. Moreover, the cycle $C_{i j}$, corresponding to the tile $\tau_{i j}$, contains the vertices $k_{i j},\left(k_{i j}+1\right), \ldots,\left(k_{i j}+i-1\right)$ and $k_{i j} \rightarrow\left(k_{i j}+i-1\right) \rightarrow\left(k_{i j}+i-2\right) \rightarrow \cdots \rightarrow\left(k_{i j}+1\right) \rightarrow k_{i j}$ (here, $u \rightarrow v$ denotes an edge directed from $u$ to $v$ ). Clearly, this is a bijection. See Figure 2 for an illustration.
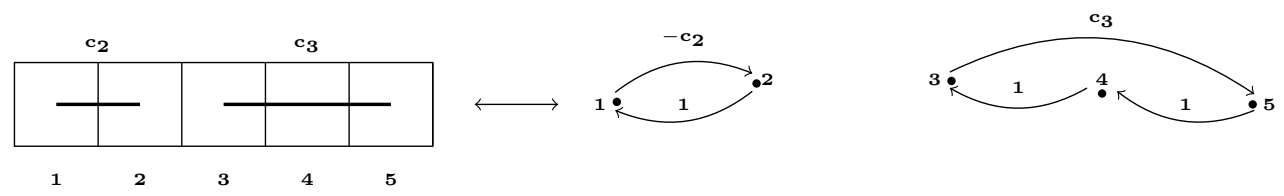

Figure 2: The bold line in each tile represents the length of the corresponding tile.

To complete the proof, we must show that this bijection preserves both the sign and weight. Suppose that $L_{\tau}$ contains $k$ cycles of even length and $m$ cycles of odd length. Let the total number of vertices in the $k$ cycles be $2 s$. Then the remaining $n-2 s$ ( $n$ is total number of vertices in $L_{\tau}$ ) vertices are among the $m$ odd cycles. Now,

$$
(-1)^{n-(k+m)} w\left(L_{\tau}\right)=(-1)^{k}(-1)^{n-m} w\left(L_{\tau}\right) .
$$

If $m$ is odd, then $n-2 s$ is odd, so $n$ is odd. If $m$ is even, then $n$ is also even. So $(-1)^{n-m}$ is always 1 . Note that only a cycle of even length contributes a negative sign towards the weight of the LSD $L_{\tau}$. Hence 
$(-1)^{n-(k+m)} w\left(L_{\tau}\right)$ is positive. From the construction of the bijection, the weight of $\tau$ is the same as the weight of the LSD $L_{\tau}$. For example, Figure 3 describes the required bijection for finding $u_{4}$ in the recurrence $u_{n}=c_{1} u_{n-1}+c_{2} u_{n-2}$.
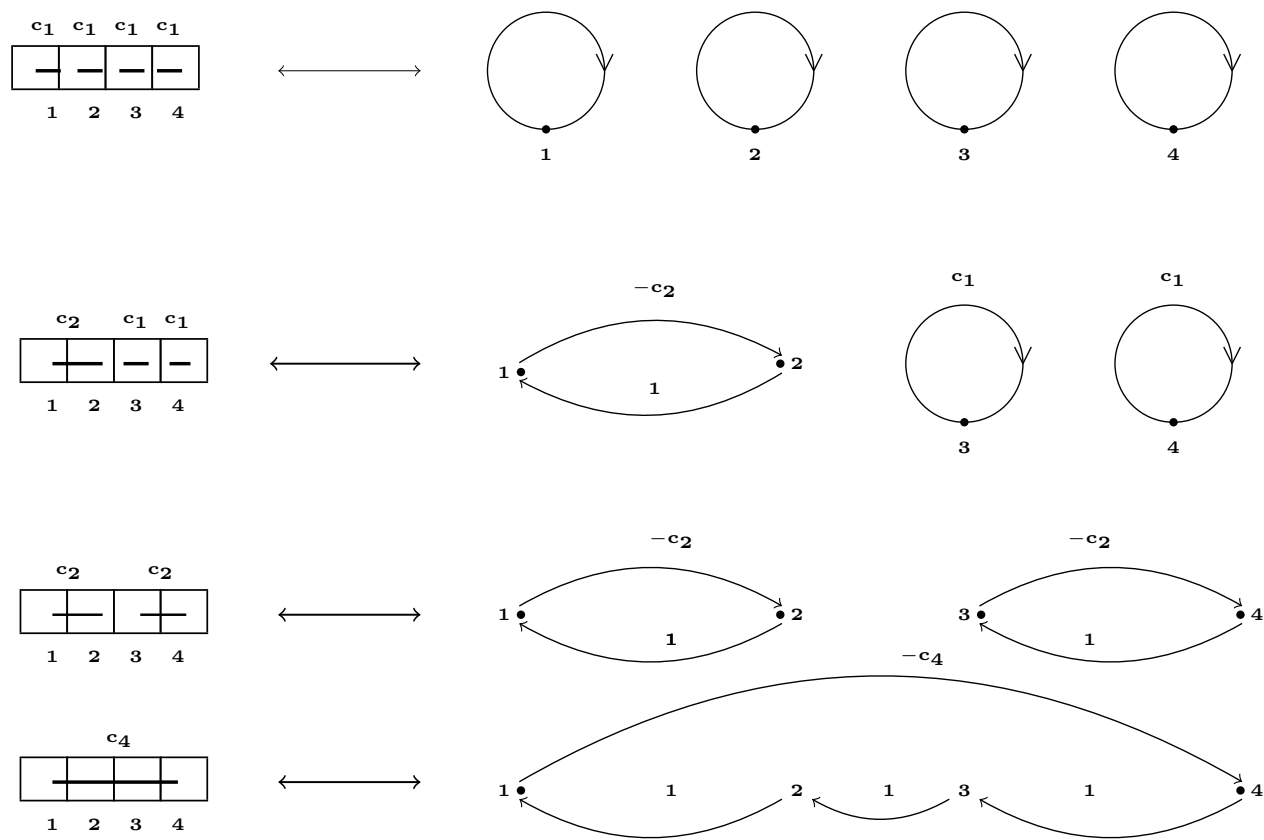

Figure 3: Each bold line in every tile represents the length of the corresponding tile. For example, the last tiling of the board contains exactly one tile of length 4 occupying the positions $1,2,3$, 4 , whereas the second tiling contains one tile of length 2 occupying the position 1,2 and two tiles of length 1 occupying the positions 3 and 4. The numbers appearing above the tiles are the weights of the corresponding tiles and the numbers appearing along the edges are the weights of the corresponding edges.

Definition 3.2. The $r$-acci sequence $F_{n}$ is defined by the recurrence

$$
F_{n}=F_{n-1}+F_{n-2}+\cdots+F_{n-r}, \quad n \geq 1,
$$

with initial conditions $F_{0}=1$ and $F_{i}=0$ for $i<0$.

One combinatorial interpretation for $F_{n}$ is the number of ways to tile a board of length $n$ using tiles of length at most $r$. In [7], the authors proved a formula for the $r$-acci numbers. Here we give a determinantal expression of the same. Consider the following $n \times n$ matrix

$$
G=\left(\begin{array}{ccccccccc}
1 & -1 & 1 & \cdots & (-1)^{r+1} & 0 & \cdots & 0 & 0 \\
1 & 1 & -1 & \cdots & (-1)^{r} & (-1)^{r+1} & \cdots & 0 & 0 \\
0 & 1 & 1 & \cdots & (-1)^{r-1} & (-1)^{r} & \cdots & 0 & 0 \\
\vdots & \vdots & \vdots & \ddots & \vdots & \vdots & \ddots & \vdots & \vdots \\
0 & 0 & 0 & \cdots & 1 & -1 & \cdots & (-1)^{n-r+1} & (-1)^{n-r+2} \\
0 & 0 & 0 & \cdots & 1 & 1 & \cdots & (-1)^{n-r} & (-1)^{n-r+1} \\
\vdots & \vdots & \vdots & \ddots & \vdots & \vdots & \ddots & \vdots & \vdots \\
0 & 0 & 0 & \cdots & 0 & 0 & \cdots & 1 & -1 \\
0 & 0 & 0 & \cdots & 0 & 0 & \cdots & 1 & 1
\end{array}\right) .
$$

Corollary 3.1. Let $F_{n}$ be the $n$-th term of the $r$-acci sequence defined above. Then $F_{n}=\operatorname{det}(G)$.

Proof. The proof follows from the combinatorial interpretation of $r$-acci numbers and the proof of Theorem 3.1 . 
Corollary 3.2 (Corollary $5[7]$ ). Let $F_{n}$ be the $n$-th term of the $r$-acci sequence. Then

$$
F_{n}=\sum_{2 i_{2}+\cdots+r i_{r} \leq n} \frac{\left(n-i_{2}-2 i_{3}-\cdots-(r-1) i_{r}\right) !}{i_{2} ! \cdots i_{r} !\left(\left(n-2 i_{2}-3 i_{3}-\cdots-r i_{r}\right) !\right)} .
$$

Proof. By Corollary 3.1, $F_{n}=\operatorname{det}(G)$ and each LSD of $D(G)$ contributes 1 to $\operatorname{det}(G)$. Again,

$$
\frac{\left(n-i_{2}-2 i_{3}-\cdots-(r-1) i_{r}\right) !}{i_{2} ! \cdots i_{r} !\left(\left(n-2 i_{2}-3 i_{3}-\cdots-r i_{r}\right) !\right)}
$$

is the number of LSD containing $\left(n-2 i_{2}-3 i_{3}-\cdots-r i_{r}\right)$ loops and $i_{t}(t=2,3, \ldots, r)$ cycles of length $t$. Hence,

$$
\operatorname{det}(G)=\sum_{2 i_{2}+\cdots+r i_{r} \leq n} \frac{\left(n-i_{2}-2 i_{3}-\cdots-(r-1) i_{r}\right) !}{i_{2} ! \cdots i_{r} !\left(\left(n-2 i_{2}-3 i_{3}-\cdots-r i_{r}\right) !\right)} .
$$

Let $f_{n}$ be the $n$-th Fibonacci number defined by

$$
f_{n}=f_{n-1}+f_{n-2}, \quad n \geq 2,
$$

with $f_{0}=f_{1}=1$. Then $f_{n}$ may be interpreted combinatorially as the number of linear tilings of a board of length $n$ using tiles of length at most 2. Consider now the $n \times n$ matrix

$$
F=\left(\begin{array}{cccccc}
1 & -1 & 0 & \cdots & 0 & 0 \\
1 & 1 & -1 & \cdots & 0 & 0 \\
0 & 1 & 1 & \cdots & 0 & 0 \\
\vdots & \vdots & \vdots & \ddots & \vdots & \vdots \\
0 & 0 & 0 & \cdots & 1 & -1 \\
0 & 0 & 0 & \cdots & 1 & 1
\end{array}\right)
$$

Then we have the following corollary.

Corollary 3.3. Let $F$ be the matrix defined as (7). Then $f_{n}=\operatorname{det}(F)$.

Proof. We use the same argument as in the proof of Theorem 3.1 above and the combinatorial interpretation for the Fibonacci numbers.

Corollary 3.4 (Binet's Formula). Let $f_{n}$ be the $n$-th Fibonacci number. Then Binet's formula says that

$$
f_{n}=\frac{\left(\frac{1+\sqrt{5}}{2}\right)^{n+1}-\left(\frac{1-\sqrt{5}}{2}\right)^{n+1}}{\sqrt{5}} .
$$

Proof. To show this, consider the matrix (4) from the proof of Corollary 2.3 above. If $x \neq y$, then the determinant of (4) can be written as $\frac{x^{n+1}-y^{n+1}}{x-y}$. Now put $x+y=1$ and $x y=-1$ in (4). Then one gets the matrix $F$. Clearly, $x$ and $y$ are the roots of the polynomial $t^{2}-t-1=0$, whence $x=\frac{1+\sqrt{5}}{2}$ and $y=\frac{1-\sqrt{5}}{2}$. By Corollary 3.3, we have $f_{n}=\operatorname{det}(F)$. By Corollary 2.3, $\operatorname{det}(F)=\frac{\left(\frac{1+\sqrt{5}}{2}\right)^{n+1}-\left(\frac{1-\sqrt{5}}{2}\right)^{n+1}}{\sqrt{5}}$, which implies the result.

\section{Determinantal Expression of the Lucas Numbers and a New Identity}

In this section, we derive a determinantal formula for members of the Lucas sequence. In the course of doing so, we first prove a new determinantal identity and, as a consequence, one obtains the desired Lucas determinantal formula. Recall that the Lucas numbers are defined recursively by

$$
\ell_{n}=\ell_{n-1}+\ell_{n-2}, \quad n \geq 2,
$$

with $\ell_{0}=2$ and $\ell_{1}=1$. Then $\ell_{n}$ can be interpreted combinatorially as the number of tilings of a circular $n$-board with tiles of length at most 2 (see [4]). 
For the variables $a$ and $b$, we consider the $n \times n(n \geq 3)$ matrix

$$
S=\left(\begin{array}{cccccc}
a+b & (-1)^{n+1} a & 0 & \cdots & 0 & b \\
(-1)^{n+1} b & a+b & a & \cdots & 0 & 0 \\
0 & b & a+b & \cdots & 0 & 0 \\
\vdots & \vdots & \vdots & \ddots & \vdots & \vdots \\
0 & 0 & 0 & \cdots & a+b & a \\
a & 0 & 0 & \cdots & b & a+b
\end{array}\right)
$$

Then the following result holds.

Theorem 4.1. Let $S$ be an $n \times n$ matrix defined as (8). Then $\operatorname{det}(S)=2\left(a^{n}+b^{n}\right)$.

Proof. We prove this theorem in the case $n=4$ (the general case can be done in a similar fashion). For $n=4$, we have

$$
S=\left(\begin{array}{cccc}
a+b & -a & 0 & b \\
-b & a+b & a & 0 \\
0 & b & a+b & a \\
a & 0 & b & a+b
\end{array}\right)
$$

It must be shown that $\operatorname{det}(S)=2\left(a^{4}+b^{4}\right)$. Regard $A=\{a, b\}$ as the set of letters. Let $C$ be the set of all cyclic words of length 4 formed by $a$ and $b$. For cyclic words, we always take the starting point to be 1 and the orientation to be clockwise. For example, Figure 4 contains three cyclic words of length 4 with starting point 1 and clockwise orientation.
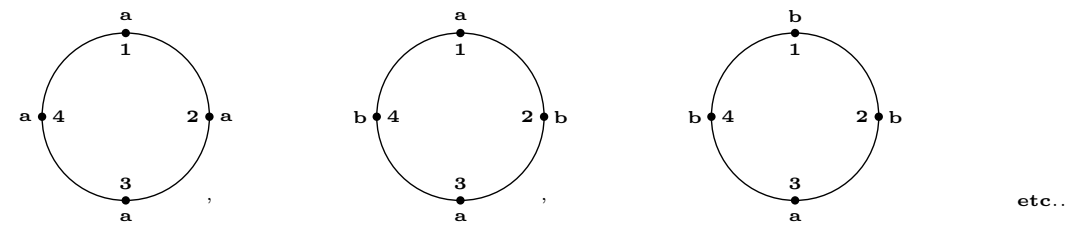

Figure 4: Illustrated are three cyclic words formed by $a, b$.

Let us introduce the following notation. We will denote by $\mathbb{Z} C(n)$ the set of all formal linear combinations
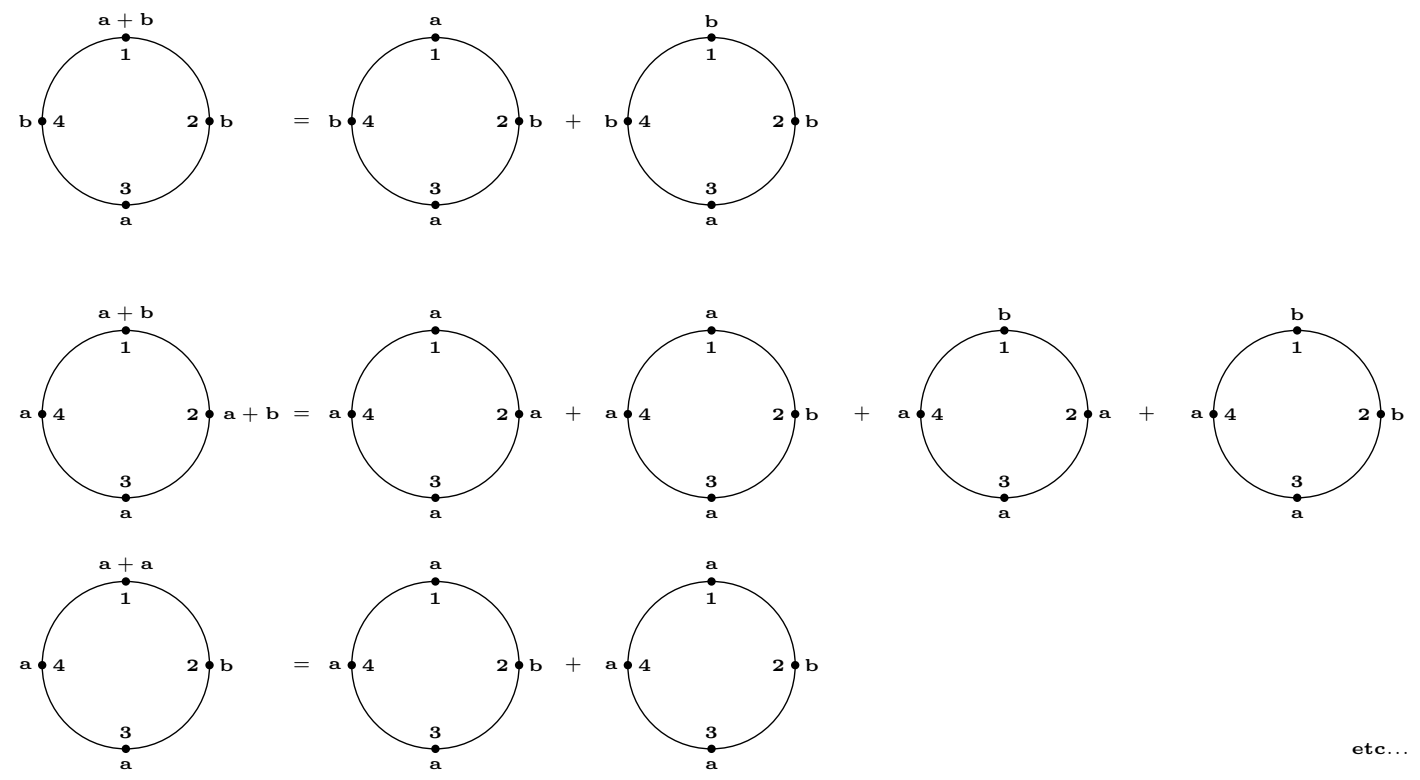

Figure 5:

of cyclic words of length $n$ (with the starting point and orientation already prescribed) with integer coefficients. For example, $c_{1}, c_{2}, c_{1}+c_{2}, 2 c_{1}-c_{2}, 0$ (the empty word) are some typical elements of $\mathbb{Z} C(n)$, where $c_{1}, c_{2}$ are cyclic words. Now let us consider Figure 5. Note that none of the items on the left side of Figure 5 is a cyclic word but may be regarded as an element of $\mathbb{Z} C(n)$ by the corresponding item on the right side. 
We wish to evaluate the sum of all possible cyclic words of length 4 formed by $a$ and $b$ such that $a b$ does not appear as a subword, i.e., $a$ and $b$ do not occur as a consecutive pair (note that $b a$ may appear as a subword). Figure 6 shows some cyclic words containing $a b$ as a subword. Let $C_{0}(\in \mathbb{Z} C(4))$ be the formal sum of all cyclic
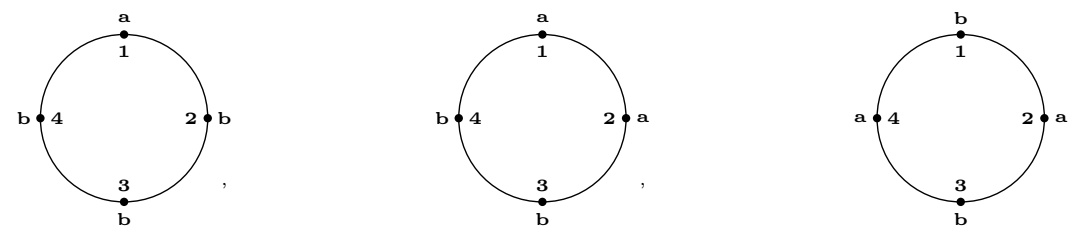

Figure 6: These are some cyclic words, where $a b$ appears as a subword. For example, in the first cyclic word, the letters $a$ and $b$ occupy the consecutive positions 1,2 , whereas the last cyclic word contains two pairs of consecutive positions 2,3 and 4,1 occupied by $a b$.

words with constant coefficient 1, i.e., $\sum_{\ell \in C} \ell$. Now the sum of all possible cyclic words of length 4 such that the

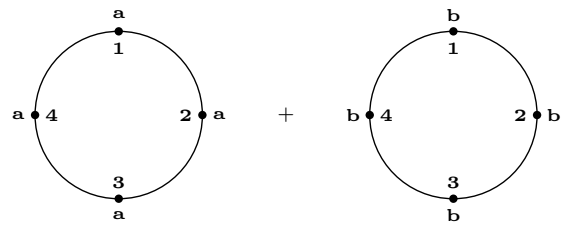

Figure 7:

letters $a$ and $b$ do not occupy two consecutive positions on the circle is clearly described in Figure 7. Again, we can calculate this sum by the PIE rule. One has that the sum of all cyclic words avoiding $a b$ as a subword is given by $C_{0}-C_{1}+C_{2}$, where $C_{1}$ is the sum of all cyclic words of length 4 formed by letters $a$ and $b$ such that

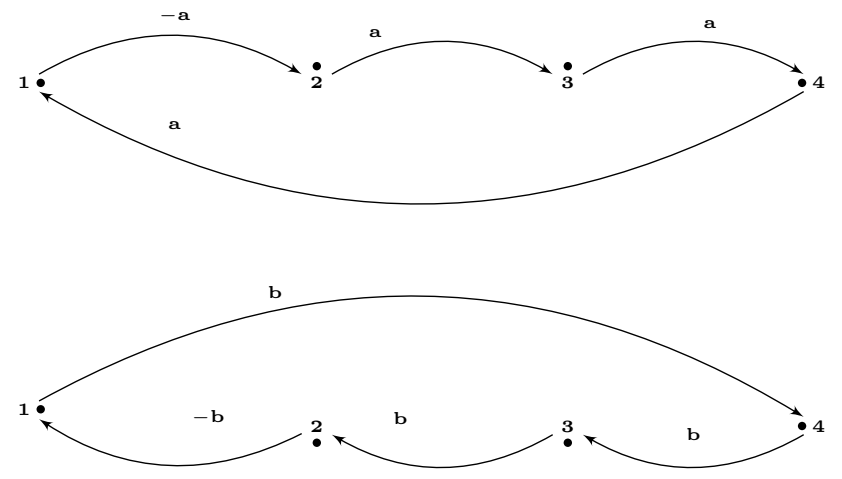

Figure 8: This figure contains two LSD of $D(S): L_{1}$ (above) and $L_{2}$ (below). The number appearing along an edge is the corresponding weight.

there is at least one pair of consecutive positions (i.e., either 1,2 or 2,3 or 3,4 or 4,1 on a circle) occupied by $a b$ (for example, see Figure 6 ) and $C_{2}$ is the sum of all cyclic words of length 4 formed by letters $a$ and $b$ such that there are at least two pairs of consecutive positions (i.e., 1,2 and 3,4 or 2,3 and 4,1) occupied by $a b$. See the last item of Figure 6 for an illustration of the latter. Now we describe a sign- and weight-preserving bijection
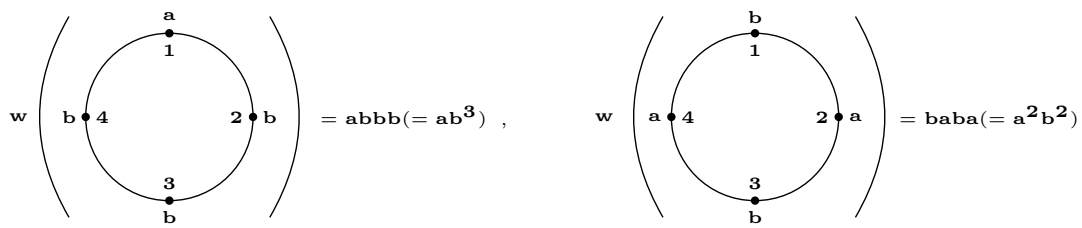

etc

Figure 9: Two cyclic words and their weights.

between the terms in $C_{0}-C_{1}+C_{2}$ and the LSDs in the set $\mathcal{L} \backslash\left\{L_{1}, L_{2}\right\}$, where $\mathcal{L}$ is the collection of all LSDs 
in $D(S)$ and $L_{1}, L_{2}$ are the LSDs in Figure 8.

The weight of a cyclic word $C$, denoted by $w(C)$, is the product (here, we mean the usual polynomial product) of all letters present in that cyclic word and extend this to $\mathbb{Z} C(n)$ by linearity, i.e., for any two cyclic words $\ell$ and $\ell, w(x \ell+y \ell):=x w(\ell)+y w(\ell), x, y \in \mathbb{Z}$. See Figures 9 and 10 for an illustration.

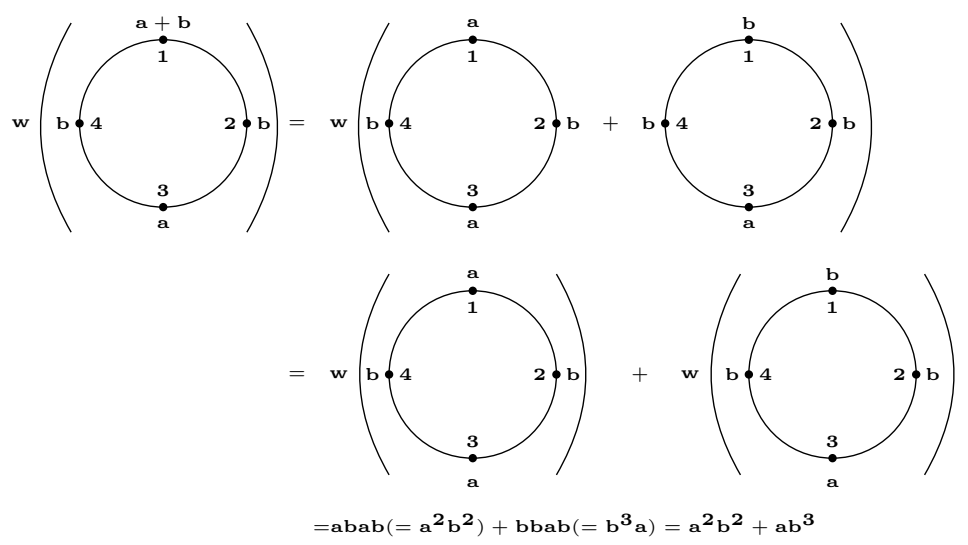

Figure 10: This figure describes the weights of the sum of cyclic words.

Figure 11 shows the required bijection, which preserves both the sign and weight. Note that $(-1)^{4-1} w\left(L_{1}\right)=$ $a^{4}$ and $(-1)^{4-1} w\left(L_{2}\right)=b^{4}$. Hence, $\operatorname{det}(S)=2\left(a^{4}+b^{4}\right)$.
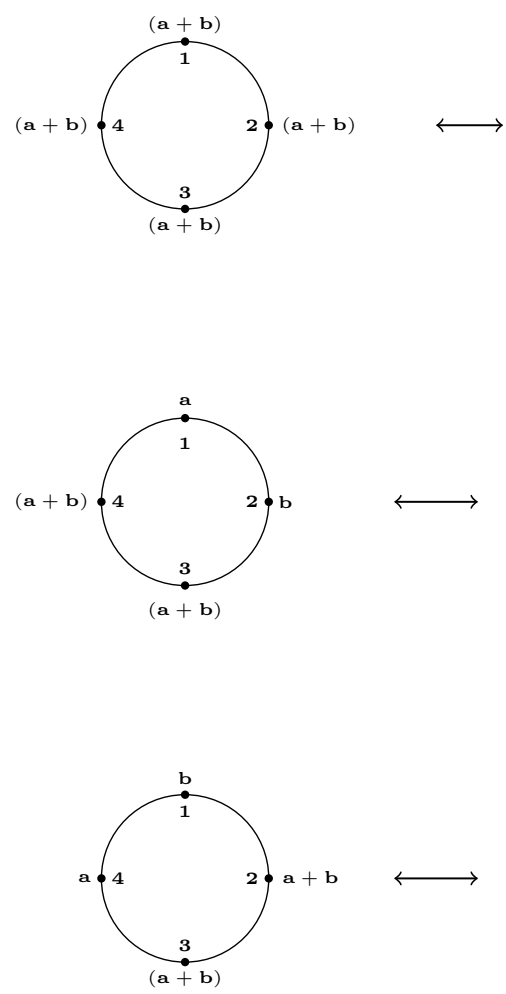
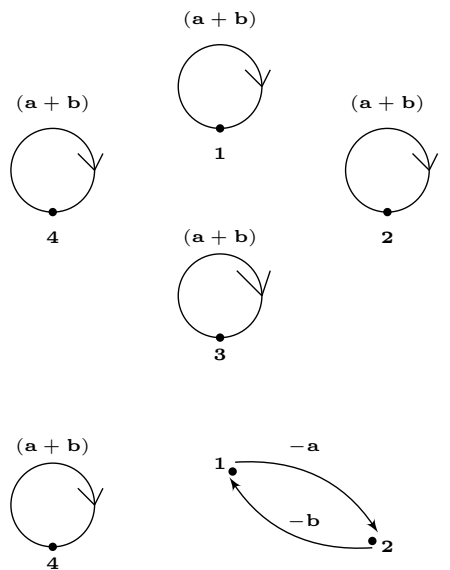<smiles>c1ccccc1</smiles>
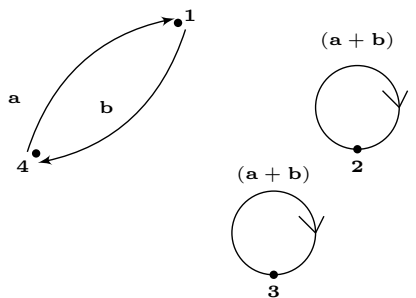

Figure 11: The number appearing along an edge in the above figure is the weight of the corresponding edge. The left-hand side of this figure describes some terms of the PIE expression.

Now we establish a determinantal expression for the Lucas numbers. First, we consider the $n \times n(n \geq 3)$ 
matrix

$$
A=\left(\begin{array}{cccccc}
1 & (-1)^{n+1} \frac{1+\sqrt{5}}{2} & 0 & \cdots & 0 & \frac{1-\sqrt{5}}{2} \\
(-1)^{n+1} \frac{1-\sqrt{5}}{2} & 1 & \frac{1+\sqrt{5}}{2} & \cdots & 0 & 0 \\
0 & \frac{1-\sqrt{5}}{2} & 1 & \cdots & 0 & 0 \\
\vdots & \vdots & \vdots & \ddots & \vdots & \vdots \\
0 & 0 & 0 & \cdots & 1 & \frac{1+\sqrt{5}}{2} \\
\frac{1+\sqrt{5}}{2} & 0 & 0 & \cdots & \frac{1-\sqrt{5}}{2} & 1
\end{array}\right) .
$$

Corollary 4.1. Let $\ell_{n}$ denote the $n$-th term of the Lucas sequence. Then $\ell_{n}=\frac{1}{2} \operatorname{det}(A)$ for $n \geq 3$.

Proof. Putting $a=\frac{1+\sqrt{5}}{2}$ and $b=\frac{1-\sqrt{5}}{2}$ in $S$, we get matrix $A$. So,

$$
\operatorname{det}(A)=2\left[\left(\frac{1+\sqrt{5}}{2}\right)^{n}+\left(\frac{1-\sqrt{5}}{2}\right)^{n}\right]
$$

On the other hand, from the proof of Theorem 4.1 and the combinatorial interpretation of the Lucas numbers, one can write

$$
\operatorname{det}(A)=\ell_{n}+\left(\frac{1+\sqrt{5}}{2}\right)^{n}+\left(\frac{1-\sqrt{5}}{2}\right)^{n},
$$

which implies the result.

\section{Acknowledgement}

I would like to thank my mentor Prof. Arvind Ayyer for his constant support, encouragement and for valuable discussion and suggestions in the preparation of this paper. Also, I would like to thank Dr. Sajal Kumar Mukherjee for many helpful discussions and for proposing Theorem 4.1. The author was supported by Department of Science and Technology grant EMR/2016/006624 and partially supported by UGC Centre for Advanced Studies. Further, the author was supported by NBHM Post Doctoral Fellowship grant 0204/52/2019/RD-II/339.

\section{References}

[1] A. Ayyer, Determinants and perfect matchings, J. Combin. Theory Ser. A 120 (2013), 304-314.

[2] A.T. Benjamin, H. Derks and J.J. Quinn, The combinatorialization of linear recurrences, Electron. J. Combin. 18(2) (2011-2), P12.

[3] A.T. Benjamin, G.M. Levin, K. Mahlburg and J.J. Quinn, Random approaches to Fibonacci identities, Amer. Math. Monthly 107 (2000), 511-516.

[4] A.T. Benjamin and J.J. Quinn, Proofs that Really Count: The Art of Combinatorial Proof, Mathematical Association of America, Washington, DC, 2003.

[5] S. Bera and S.K. Mukherjee, Combinatorial proofs of some determinantal identities, Linear and Multilinear Alg. 66 (2018), 1659-1667.

[6] A.R. Brualdi and D. Cvetkovic, A Combinatorial Approach to Matrix Theory and its Application, Discrete Mathematics and Its Applications, CRC Press, Boca Raton, London, New York, 2009.

[7] J. McLaughlin and B. Sury, Powers of a matrix and combinatorial identities, Integers 5(1) (2005), \#A13.

[8] S.K. Mukherjee and S. Bera, Combinatorial proofs of the Newton-Girard and Chapman-Costas-Santos identities, Discrete Math. 342 (2019), 1577-1580.

[9] B.E. Sagan, The Symmetric Group Representations, Combinatorial Algorithms, and Symmetric Functions, Graduate Texts in Mathematics, Springer-Verlag, New York, 2001.

[10] M. Shattuck, Recounting some reciprocal Fibonacci number relations, Util. Math. 79 (2009), $259-265$.

[11] B. Sury, A curious polynomial identity, Nieuw Arch. Wisk. 11 (1993), 93-96.

[12] D. Zeilberger, A combinatorial proof of the Newton's identity, Discrete Math. 49 (1984), 319. 